\title{
Los sistemas complejos como herramienta para la sustentabilidad. Una visión desde la perspectiva de la Teoría Crítica
}

\author{
Salvador Peniche Camps ${ }^{1}$ \\ "La ciencia no puede resolver el misterio último de la naturaleza. Y esto es por- \\ que en el último análisis, nosotros somos parte de la naturaleza y, por lo tanto, \\ parte del misterio que estamos tratando de resolver" \\ Max Planck
}

\section{Resumen}

El objetivo del ensayo que se presenta consiste en discutir la naturaleza de la metodología de los sistemas complejos como herramienta útil para el análisis y la gestión de la sustentabilidad. Se concluye que la construcción de un modelo de sistemas complejos socialmente sensibles es un objetivo pendiente y urgente para enfrentar el colapso ambiental que sufre la sociedad contemporánea.

Palabras clave: metodología de análisis para la sustentabilidad, teoría de la ciencia.

\section{Introducción}

Planteamos un contexto crítico sobre los alcances y limitaciones de la herramienta metodológica de los sistemas complejos, entendidos como un instrumento útil para abordar el colapso socioambiental que vive la humanidad en nuestros tiempos.

Nos proponemos contextualizar el modelo de análisis e intervención que ofrecen los sistemas complejos en uno de los debates más significativos sobre el acceso al

Fecha de Recepción: 18 de Octubre de 2020. Fecha de Aceptación: 10 de Diciembre de 2020.

1 Profesor e Investigador. Departamento de Economía. CuCEA. Universidad de Guadalajara. Miembro del Sistema Nacional de Investigadores (SNI) Nivel I. Orcid ID: https://orcid.org/0000-0001-84904178. Correo electrónico: peniche@hotmail.com 
conocimiento científico, es decir, la confrontación epistemológica entre el neopositivismo o positivismo lógico y la Teoría Crítica.

Concluimos que la utilidad del modelo de sistemas complejos es incuestionable, sin embargo, su potencia, la posibilidad de su trasformación de metáfora analítica a instrumento de política pública para la sustentabilidad depende de trascender el enfoque neopositivista que lo ha caracterizado en la práctica y los ámbitos académicos.

\section{Alcances de la sistémica compleja como instrumento para la investigación científica y la intervención: colapso ambiental y ecología profunda.}

Un sistema es un conjunto de elementos que se relacionan entre sí, generando un comportamiento emergente. La historia de la perspectiva de sistemas es muy fecunda e inicia formalmente con los planteamientos de Bertalanffy quien propuso, a mediados del siglo pasado, el edificio lógico conceptual de la sistémica. El legado del biólogo alemán consiste en haber planteado de manera rigurosa la idea de la transdisciplina y la apertura metodológica como precondiciones para entender la vida y los ecosistemas.

"De aquí que adelantemos una nueva disciplina llamada Teoría general de los sistemas. Su tema es la formulación y derivación de aquellos principios que son válidos para los «sistemas» en general. El sentido de esta disciplina puede ser circunscrito como sigue. La física se ocupa de sistemas de diferentes niveles de generalidad. Se dilata desde sistemas bastante especiales como los que aplica el ingeniero a la construcción de un puente o una máquina, hasta leyes especiales de disciplinas físicas como la mecánica o la óptica, y hasta leyes de gran generalidad, como los principios de la termodinámica, aplicables a sistemas de naturaleza intrínsecamente diferente --mecánicos, calóricos, químicos o lo que· sean.

Nada prescribe que tengamos que desembocar en los sistemas tradicionalmente tratados por la física. Podemos muy bien buscar principios aplicables a sistemas en general, sin importar que sean de naturaleza física, biológica o sociológica. Si planteamos esto y definimos bien el sistema, hallaremos que existen modelos, principios y leyes que se aplican a sistemas generalizados, sin importar su particular género, elementos y $<<$ fuerzas $>>$ participantes.

La consecuencia de la existencia de propiedades generales de sistemas es la aparición de similaridades estructurales o isomorfismos en diferentes campos (Bertalanfyy, 1989:33)

Los sistemas complejos se diferencian radicalmente de los sistemas mecánicos emanados de la ciencia clásica. Las máquinas "newtonianas", pueden ser consideradas sistemas jerárquicos y ordenados que producen resultados predecibles. Tal es el caso de un automóvil o una computadora los cuales pueden ser vistos como sistemas mecánicos con estructuras complicadas. En cambio, en los sistemas complejos, los elementos no se ordenan de manera jerárquica y son impredecibles. El clima, entendido como un sistema complejo cásico, puede referirnos a la probabilidad de lluvias en un territorio, pero no sobre su lugar o intensidad exactos. 
Los problemas socioambientales de la actualidad son, por antonomasia, sistemas complejos por ser inciertos y no jerárquicos. Debido a que no es posible entender el funcionamiento de un sistema complejo con visiones parciales o mecánicas, la incorporación de la enseñanza y práctica de la metodología de los sistemas complejos se vuelve una prioridad social.

Según García:

"Un sistema complejo es una representación de un recorte de esa realidad, conceptualizado como una totalidad organizada (de ahí la denominación de sistema), en la cual los elementos no son separables y por lo tanto no pueden ser estudiados aisladamente" (García, 2006: 21).

El colapso ambiental que vive la humanidad es el resultado del comportamiento emergente del sistema que se ha construido a lo largo de la evolución del sistema capitalista. El proceso, que en su etapa actual ha recibido la denominación de "La gran aceleración", se ha acercado a la etapa terminal. Aspectos fundamentales que permiten el mantenimiento de la vida en el sistema "Planeta Tierra", como la biodiversidad, el ciclo del nitrógeno o el cambio climático, parecen haber cruzado el umbral de la recuperación, del punto de no retorno.

La gran aceleración puede definirse como una etapa característica del Antropoceno (era geológica que se caracteriza por el efecto transformador de la sociedad humana):

"El crecimiento exponencial de las actividades humanas está aumentando la preocupación de que una mayor presión sobre la Tierra. El sistema podría desestabilizar la biofísica crítica sistemas y desencadenan abruptos o irreversibles cambios ambientales que serían perjudiciales o incluso catastrófico para el bienestar humano. Esto es un profundo dilema porque el predominante paradigma de desarrollo social y económico permanece en gran parte ajeno al riesgo de desastres ambientales inducidos por humanos en continentes y escalas planetarias" (Stern 2007). (Citado de Rockstrom, et al, 2009:32)

La idea detrás del imperativo de la utilización de la sistémica compleja consiste en entender el funcionamiento del sistema "sociedad-medio ambiente" para que, con la ayuda de ese conocimiento, se intente revertir o atemperar deterioro acelerado y permanente. Para ello, es menester situar en el centro de la cultura en su sentido más amplio, el concepto del colapso y el riesgo de la destrucción de los principales sistemas de soporte de la vida en el planeta y considerar la posibilidad de la extinción de la especie. Posicionar el imperativo de la supervivencia de la sociedad humana como prioridad impostergable en la vida pública, en la academia y en los programas de gobierno, es lo que Capra llama la adopción de la "ecología profunda".

"El nuevo paradigma podría denominarse una visión holística del mundo, ya que lo ve como un todo integrado más que como una discontinua colección de partes. También podría llamarse una visión ecológica, usando el término "ecológica» en un sentido mucho más amplio y profundo de lo habitual. La percepción desde la ecología profunda reconoce 
la interdependencia fundamental entre todos los fenómenos y el hecho de que, como individuos y como sociedades, estamos todos inmersos en (y finalmente dependientes de) los procesos cíclicos de la naturaleza. (Capra, 1996: 28).

\section{Positivismo lógico versus teoría crítica: el contexto del debate sobre la metáfora de los sistemas}

La teoría de sistemas no es una "receta" para la solución de los problemas socio-ambientales. Se concibe más bien como una metáfora, una visión ideal del funcionamiento de los sistemas complejos que conforman la realidad objetiva. Sin embargo, en la práctica, el incremento de la complejidad de los modelos y el gran avance de la matemática utilizada en su construcción han revividlo el debate añejo entre el positivismo lógico o neopositivismo y la Teoría Crítica en torno a las posibilidades teleológicas de la ciencia en general y de esta herramienta en particular.

El positivismo lógico constituye un movimiento filosófico que pone énfasis en las capacidades cognitivas del intelecto y señala que el conocimiento sólo puede ser resultado de la práctica de la razón y la aplicación del método científico. Desde esta perspectiva, la comprobación empírica es el único criterio de la verdad.

"Las observaciones que hacemos en la vida cotidiana, así como las observaciones más sistemáticas de la ciencia, revelan ciertas repeticiones o regularidades en el mundo. El día siempre sigue a la noche; las estaciones del año se repiten en el mismo orden; el fuego siempre se siente caliente; los objetos caen cuando los soltamos, y así sucesivamente. Las leyes de la ciencia no son más que declaraciones que expresan estas regularidades de manera tan precisa como sea posible. (Carnap, S/A: 5)

Los filósofos del Círculo de Viena entre los que se encuentran Rudolf Carnap, Otto Neurath y Kurt Goeddel plantearon en los años 20 del siglo pasado, los principales axiomas del positivismo lógico con lo cual, desde los campos de las ciencias duras, se apartaron de cualquier manifestación no empírica, adoptando una posición mecanicista en la interpretación del funcionamiento de los sistemas. Los filósofos del círculo de Viena consideraban que existía una verdad "objetiva", es decir, independiente de intereses o ideologías y que esta era asequible a través del método científico.

Por su parte, la Teoría Crítica, acercamiento filosófico de la ciencia propuesto por intelectuales pertenecientes a la llamada Escuela de Frankfort (Theodor Adorno, Max Horkheimer y Herbert Marcuse), desarrollaron una dura la crítica a la moral y la cultura occidental. Para la escuela de Frankfort, la ciencia viene determinada por la estructura social que la contiene, por lo que la objetividad científica es imposible.

"En la realidad social, a pesar de todos los cambios, la dominación del hombre por el hombre es todavía la continuidad histórica que vincula la Razón pre-tecnológica con la tecnológica. Sin embargo, la sociedad que proyecta y realiza la transformación tecnológica de la naturaleza, altera la base de la dominación, reemplazando gradualmente la dependencia personal (del esclavo con su dueño, el siervo con el señor de la hacienda, el señor 
con el donador del feudo, etc.) por la dependencia al «orden objetivo de las cosas» (las leyes económicas, los mercados, etc.). Desde luego, el «orden objetivo de las cosas» es en sí mismo resultado de la dominación, pero también es cierto que la dominación genera ahora una racionalidad más alta: la de una sociedad que sostiene su estructura jerárquica mientras explota cada vez más eficazmente los recursos mentales y naturales y distribuye los beneficios de la explotación en una escala cada vez más amplia. Los límites de esta racionalidad, y su siniestra fuerza, aparecen en la progresiva esclavitud del hombre por parte de un aparato productivo que perpetúa la lucha por la existencia y la extiende a una lucha internacional total que arruina las vidas de aquellos que construyen y usan este aparato. (Marcuse, 1968: 171)

El planteamiento de los teóricos críticos nos lleva a la discusión sobre el alcance y las limitaciones de la metodología de los sistemas complejos pues pone en el centro del debate no sólo la potencia de sus conclusiones sino la metodología de su construcción.

Así, el análisis del impacto de la sistémica en la construcción de la sustentabilidad se ha constituido como un campo fértil para la objetivación del debate epistemológico entre las dos escuelas filosóficas. La interpretación convencional de la sistémica en amplios sectores académicos, su fuerte formalización asentada en las posibilidades que ofrece la cibernética y la WWW, ha fortalecido la visión positivista hasta el grado de posicionarla como la dominante.

En la práctica de la política pública y en la academia, las posiciones se dividen entre aquellos metodólogos que estudian las posibilidades formales de la herramienta, la capacidad de establecer vínculos causales y futuros (la modelización de la realidad objetiva) y aquellos que enfatizan el carácter socialmente determinado tanto de las premisas, como de la construcción y funcionamiento de los modelos.

La construcción de una ciencia de la sistémica desde la perspectiva de la complejidad sugiere la profundización de la exploración teórica expuesta con el objetivo de establecer un claro deslinde de posiciones mecanicistas que limitan su utilidad. La objeción de la Teoría Crítica a las posiciones neopositivistas en el sentido del establecer un divorcio entre el "sujeto que conoce y el objeto conocido" es hoy más pertinente que nunca y se plantea como el más importante obstáculo de la utilidad de la sistemática en la política pública. El objetivo es evitar la inocuidad de clase que sugiere el neopositivismo en la práctica de la ciencia en general y en la utilización de los sistemas complejos en particular.

La construcción de un modelo sistémico socialmente sensible es una tarea pendiente y en construcción. Se podría pensar que, dado el colapso ambiental y el riesgo real de la desaparición de la especie, se generarían condiciones políticas e ideológicas propicias.

Lo que se observa es, sin embargo, el desmembramiento del tejido social a escala global. La hipótesis avanzada por Mossadeq nos habla de las repercusiones sociales del agotamiento de las reservas de petróleo barato.

"Esta inevitable transición energética de los combustibles fósiles de alta calidad a formas de energía más caras y de calidad, que se completarán mucho antes de la a finales de este 
siglo, y posiblemente mucho antes, forzará un cambio de paradigma en la organización de la civilización. El siglo XXI, en este contexto, es fundamental para la humanidad a medida que la civilización industrial pivota a través de un proceso de transición, impulsada por la compleja interacción entre las sociedades humanas y las realidades biofísicas" (Mosaddeq, 2017: 1)

La disyuntiva que enfrenta la sociedad contemporánea está en escoger uno de los dos caminos que plantea el colapso socioambiental y decidir su derrotero. $\mathrm{O}$ se construye un sistema autopoyético, es decir se socializa una visión eco centrista en donde el papel de la sociedad sea determinado por los límites biofísicos existentes en el sistema mundo o se continúa con el modelo actual caracterizado por la lucha tribal por la supervivencia, en condiciones de escases acelerada de recursos y trasgresión de los límites biofísicos del desarrollo.

Como líneas estratégicas de trabajo para avanzar en la dirección de la construcción de una metodología sistémica socialmente sensible y útil en la tarea de la construcción de la primera estrategia, se podría pensar inicialmente en dar mayor peso específico a la incertidumbre existente en el tipo de relación de los elementos integrantes del sistema y entre sus actores, es decir, desarrollar modelos de evaluación cualitativa de impactos socioambientales y la incorporación de la valoración subjetiva de las resistencias políticas e ideológicas.

\section{Conclusiones}

La sistémica constituye una poderosa herramienta para abordar los graves problemas socioambientales que caracterizan a la sociedad contemporánea. El origen del aumento en flecha de la importancia de los sistemas complejos como instrumento de análisis y gestión socioambiental consiste en la aceleración del deterioro socioambiental y el fracaso de los modelos existentes para enfrentarlo. Lo conducente, sugerimos, es entender y difundir la idea de la realidad del colapso socioambiental y, consecuentemente, comunicar la imperiosa necesidad de abordar la problemática con un instrumento que permita entender las relaciones complejas entre los factores que dan como resultado el colapso ambiental.

Sin embargo, la práctica de la sistémica en la actualidad se caracteriza por una interpretación neopositivista que sostiene la objetividad de los modelos, tanto en los principios en los que se basa su construcción, como en los mecanismos que posibilitan su funcionamiento y, lo más importante, la interpretación de conclusiones que llevan a la elaboración de las políticas socioambientales.

Con la visión de la de la Teoría Crítica se pretende incorporar el factor social e ideológico en el análisis y reconocer la imposibilidad de la objetividad dados los condicionamientos sociales de cualquier planteamiento científico. 


\section{Referencias bibliográficas}

Bertalanffy, L. (1989). Teoría General de los sistemas. Fundamentos, desarrollo, aplicaciones. México: FCE.

Carnap, R. (S/A). Una introducción a la filosofía de la ciencia. http://bibliotecadigital. ilce.edu.mx/Colecciones/ReinaCiencias/_docs/Introduccion_filosofia_ciencia.pdf

Capra, F. (1996). La trama de la vida. Una nueva perspectiva $\overline{\text { de }}$ los sistemas vivos. España: Anagrama.

García, R. (2006). Sistemas complejos. Conceptos, métodos y fundamentación epistemológica de la investigación interdisciplinaria. España: Gedisa

Marcuse, H. (1968). El hombre unidimensional. Ensayo sobre la ideología de la sociedad industrial avanzada. España: Planeta

Mossadeq, N. (2017). Failing states. Collapsing systems. Biophysical triggers of political violence. EE:UU: Springer

Stromberg, et al (2009). Planetary boundaries: exploring the safe operating system of humanity. Ecology and society. 14(2). 